
(клинические наблюдения)

\author{
Зельтер П.М. ${ }^{\text {* }}$, Колсанов А.В. ${ }^{1}$, Соловов Д.В. ${ }^{1}$, Капишников А.В. ${ }^{1}$, Пекшева М.С. ${ }^{2}$ \\ 1 ГБОУ ВПО “Самарский государственный медицинский университет” Минздрава России, Самара, Россия \\ ${ }^{2}$ СПб ГБУЗ “Городская больница Святой преподобномученицы Елизаветы”, Санкт-Петербург, Россия
}

\title{
The use of CT in diagnostics of vascular-ureteral conflict (clinical observations)
}

\author{
Zelter P.M. ${ }^{1}$, Kolsanov A.V. ${ }^{1}$, Solovov D.V. ${ }^{1}$, Kapishnikov A.V. ${ }^{1}$, Peksheva M.S. ${ }^{2}$ \\ ${ }^{1}$ Samara State Medical University, Samara, Russia \\ ${ }^{2}$ Saint-Petersburg St.Elisabeth state hospital, Saint-Petersburg, Russia
}

Цель исследования: изучить различные способы диагностики сосудисто-лоханочно-мочеточникового конфликта при помощи КТ и их возможность для применения в клинической практике.

Материал и методы. Изучены методики сплитболюсного контрастирования, компьютерной обработки и последующего трехмерного (3D) моделирования для совмещения фаз болюсного исследования, приведены данные трех пациентов.

Результаты. Сплит-болюсное контрастирование позволило на одной серии изображений оценить соотношение чашечно-лоханочной системы и артерий, но методика такого исследования не является четко определенной, зависит от учреждения и от состояния пациента. 3D-моделирование является более универсальной методикой, представляет собой автоматическую постпроцессинговую обработку стандартного исследования мочевыводящей системы.

Заключение. Применение обеих методик позволяет достоверно визуализировать компрессию чашечнолоханочной системы и мочеточника аномальными сосудами, исключить иные признаки гидронефроза.

Ключевые слова: компьютерная томография, гидронефроз, 3D-моделирование, сплит-болюс.

Ссылка для цитирования: Зельтер П.М., Колсанов А.В., Соловов Д.В., Капишников А.В., Пекшева М.С. Способы диагностики сосудисто-лоханочно-мочеточникового конфликта с помощью компьютерной томографии (собственные наблюдения из практики). Медицинская визуализация. 2019; 23 (2): 76-81. DOI: 10.24835/1607-0763-2019-2-76-81.
Purpose. To study various ways of diagnosing a vascular-pelvic-ureteral conflict using CT and the abillity to use it in routine work.

Materials and methods. Split-bolus contrastenhanced CT, and computer processing and subsequent three-dimensional modeling have been studieds, data from three patients are presented.

Results. Split-bolus contrasting allowed to estimate the ratio of the renal pelvis system and arteries in one series of images, but the methodology of this study is not specific, depends on the institution and on the patient's condition. Three-dimensional modeling is a more universal technique; it is postprocessing of standard urinary tract examination.

Findings. The use of both techniques allows to reliably visualize the compression of collecting system and ureter by abnormal vessels, and to eliminate other signs of hydronephrosis.

Key words: computed tomography, hydronephrosis, 3D modeling, split-bolus contrast-enhancement.

Recommended citation: Zelter P.M., Kolsanov A.V., Solovov D.V., Kapishnikov A.V., Peksheva M.S. The use of CT in diagnostics of vascular-ureteral conflict (clinical observations). Medical Visualization. 2019; 23 (2): 76-81. DOI: 10.24835/1607-0763-2019-2-76-81.

$\star \star \star$

\section{Введение}

Гидронефроз - расширение чашечно-лоханочной системы (ЧлС) почки любой этиологии. Гидронефроз является актуальной проблемой 
современной урологии, требующей взвешенного подхода в диагностической тактике ввиду вариабельности причин, его вызвавших [1]. Причинами гидронефроза могут являться в том числе и "добавочные" сосуды, высокое отхождение мочеточника, нефроптоз, мочекаменная болезнь, воспаление или увеличение забрюшинных лимфатических узлов, опухоль толстой кишки, опухоль лоханки, аневризма аорты и т.д. Тактически целесообразно рассматривать гидронефроз как осложнение конкретного заболевания. Широкое распространение компьютерной томографии (КТ) позволило оптимизировать диагностическую тактику в ведении сложных пациентов с неустановленной причиной расширения ЧЛС [2].

Сосудисто-лоханочно-мочеточниковый конфликт (СЛМК) - состояние, при котором отмечается близкое расположение элементов члС и почечных сосудов и которое приводит к гидронефрозу или гидрокаликозу [3]. К сожалению, анализ статистики различных причин гидронефроза и распространенности СЛМК в Российской Федерации не ведется, и сказать достоверно, у скольких людей обструкция мочеточника вызвана именно конфликтами, не представляется возможным. Тем не менее установлено, что значительную часть причин гидронефрозов и гидрокаликозов (до 30\%) составляют конфликты между стенками члС и почечными сосудами [4]. Конфликт может происходить на разных уровнях: шейки чашечки, лоханки или мочеточника.

Аномальные артерии, которые могут вызывать обструкцию мочевых путей, имеют различное происхождение: отходящие от магистральной почечной артерии к полюсам или отдельные стволы. Было установлено, что аномальные почечные артерии встречаются у 20-35\% людей. Такие артерии отходят от аорты, art. suprarenalis, iliaca communis, ilaca externa et interna, art. sacralis media, phrenica, mesenterica inferior, hepatica dextra и т.д. у 8\% людей наблюдается рассыпной тип строения почечной артерии, при котором разделение происходит в непосредственной близости от аорты [5].

Не любая сосудистая аномалия вызывает нарушение оттока мочи. Наиболее часто ситуация подчиняется так называемому закону Екегорна: механическое препятствие для оттока мочи и развитие гидронефроза происходит в том случае, если аномальный сосуд проходит позади мочеточника (лоханки) и направляется к передней поверхности или наоборот, т.е. происходит перекрест. Также мочеточник или шейка чашечки может оказаться в сосудистом "пинцете" из артерий, артерии и вены или двух вен, чаще варикозно измененных [6].

\section{Цель исследования}

Проанализировать результаты использования двух методик КТ: сплит-болюсного введения контрастного вещества и виртуального 3D-моделирования с помощью сегментации при диагностике возможного уретеровазального конфликта.

\section{Материал и методы}

Были проанализированы данные исследований трех пациентов с неустановленной причиной расширения ЧЛС. Всем пациентам проводилась КТ с болюсным контрастированием, у двоих из них методика была модифицирована для получения смешанной фазы.

Сплит-болюс, или раздельное болюсное введение контрастного вещества, - методика, при которой пациенту вводят контрастное вещество за несколько минут до выполнения артериальной и венозной фаз. Существует несколько модификаций сплит-болюсного исследования: L.C. Chow и соавт. [7] использовали следующую методику: после нативного сканирования 40 мл контрастного вещества вводилось через шприц-инжектор со скоростью 2 мл/с, затем через 4 мин вводилось еще 80 мл с той же скоростью и через 120 с выполнялась совмещенная нефрографическая и выделительная фазы. Такая методика оказалась высокоэффективной в выявлении уротелиальных опухолей и почечно-клеточного рака. Для оценки сосудистой анатомии В. Sauer и соавт. [8] рекомендовали следующий подход: нативное исследование, затем введение 60 мл контрастного вещества вручную с последующим введением 20 мг фуросемида. Артериальная и венозная фазы выполнялись через 10 мин с введением контрастного вещества (объем его не оговаривается) с помощью шприца-инжектора. Подобная методика позволяла с высокой точностью, подтвержденной интраоперационно, определять аномалии как артериальной, так и венозной анатомии.

\section{Результаты}

В качестве основы для сплит-болюсного контрастирования мы взяли методику В. Sauer и соавт., но без введения фуросемида. Объем введенного до болюсного исследования контрастного вещества был сокращен до 20 мл. Такой объем был подобран эмпирически и, учитывая большую длительность ожидания фактически выделительной фазы, на наш взгляд, является достаточным. Приведем несколько клинических примеров.

\section{Клинический пример 1}

Пациент А., 19 лет, из анамнеза известно, что гидроуретеронефроз был выявлен несколько лет назад при 


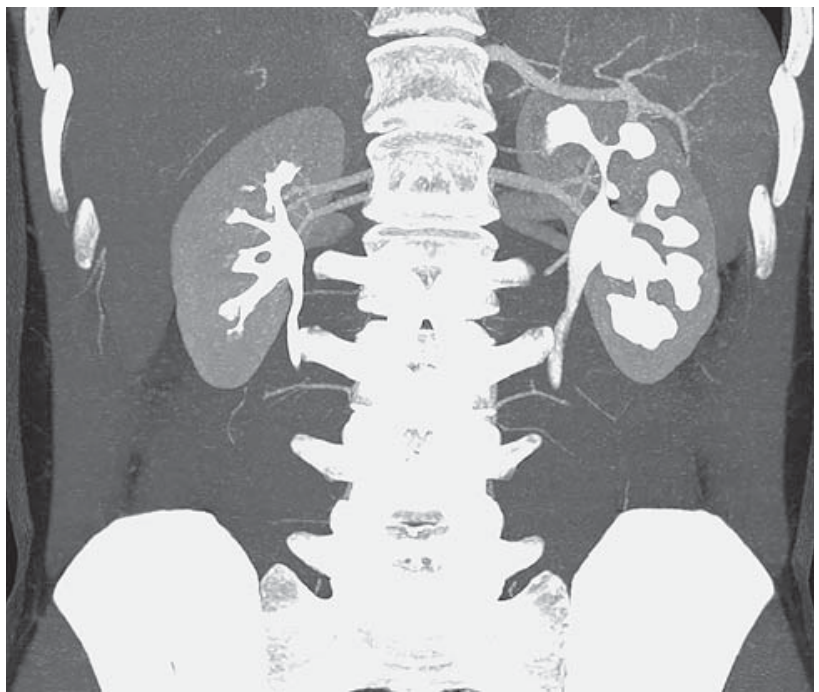

Рис. 1. КТ, артериальная фаза, корональная реконструкция, режим MIP. Справа отмечается удвоение почечной артерии.

Fig. 1. CT, arterial phase, coronal reconstruction, MIP mode. On the right there is a doubling of the renal artery.

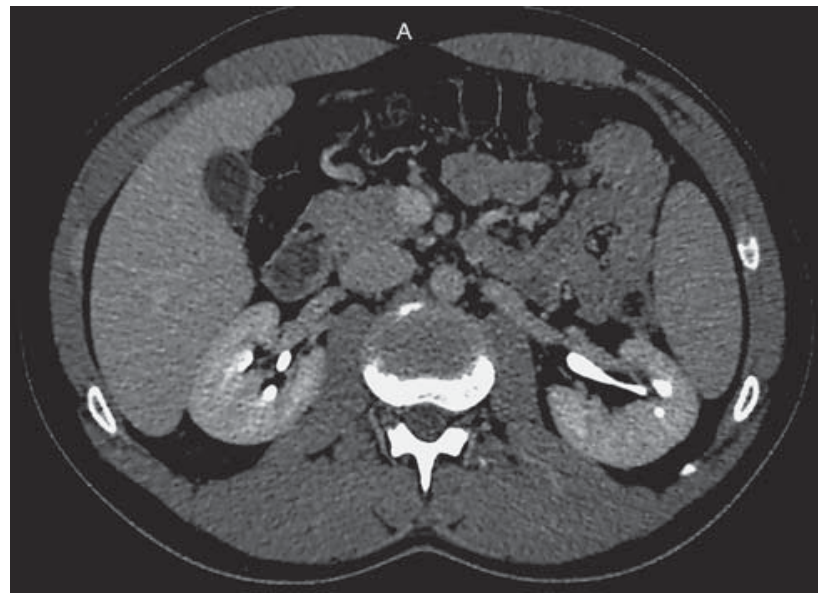

Рис. 2. КТ, венозная фаза, аксиальная плоскость. С обеих сторон визуализируются единичные почечные вены.

Fig. 2. CT, venous phase, axial plane. Single renal veins are visualized on both sides.

ультразвуковом исследовании. Для исключения сосудистой обструкции был направлен на КТ с контрастированием. Выполнена нативная фаза, затем вручную введено 20 мл раствора йогексола 350 мг/мл. Через 10 мин введено 80 мл йогексола 350 мг/мл со скоростью 4 мл/с. При анализе артериальной фазы отмечается аномалия артериального кровоснабжения: справа верхний полюс и средний отдел кровоснабжают две артерии крупного диаметра, а нижний - более мелкая артерия (рис. 1). Слева отмечается добавочная артерия верхнего полюса малого диаметра. Но очевидно, что артерия проходит

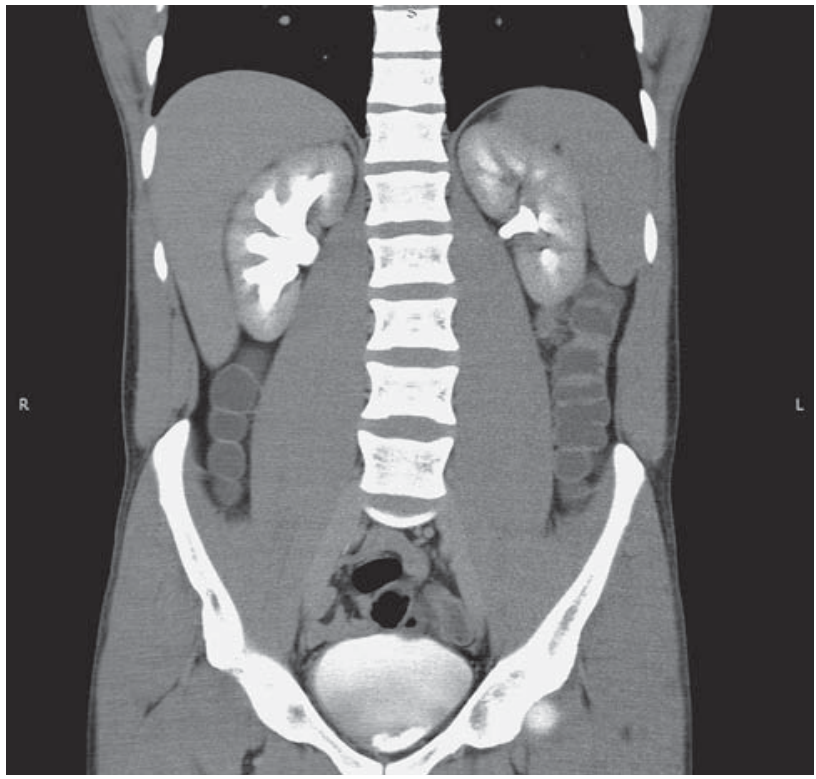

Рис. 3. КТ, выделительная фаза болюсного исследования, корональная плоскость. Справа отмечается расширение ЧЛС.

Fig. 3. CT, excretory phase of the bolus enhanced study, the coronal plane. The dilatation of CLS is seen on the right.

не в области шейки чашечки, а расширение чашечнолоханочной системы распространяется дистальнее перекреста с сосудом.

При анализе венозной фазы отмечается классический вариант строения с отхождением единичных почечных вен от нижней полой вены (рис. 2). Таким образом, диагноз сосудисто-чашечно-лоханочного конфликта был исключен, основным рассматриваемым фактором осталось врожденное расширение мочевых путей.

\section{Клинический пример 2}

Пациент Т., 26 лет, обратился к урологу с жалобами на тянущие боли в поясничной области справа. Из анамнеза известно, что ему выставлен диагноз хронический пиелонефрит. В настоящее время отклонений в общем анализе крови и мочи не выявлено. Направлен на КТ для исключения обструкции, в первую очередь мочекаменной болезни. В нативной фазе конкрементов в мочевых путях не выявлено, отмечается расширение члс (рис. 3). Было проведено стандартное исследование почек и мочевыделительной системы с болюсным контрастированием. Введено 100 мл йогексола 350 мг/мл со скоростью 4 мл/с, выполнялись артериальная, венозная и выделительная фазы (на 10-й минуте).

В артериальной фазе справа определяется рассыпной тип строения почечной артерии (рис. 4), но достоверно конфликта без контрастирования ЧлС не определяется.

Было выполнено постпроцессинговое 3D-моделирование данных КТ. Для этого использовалась про- 


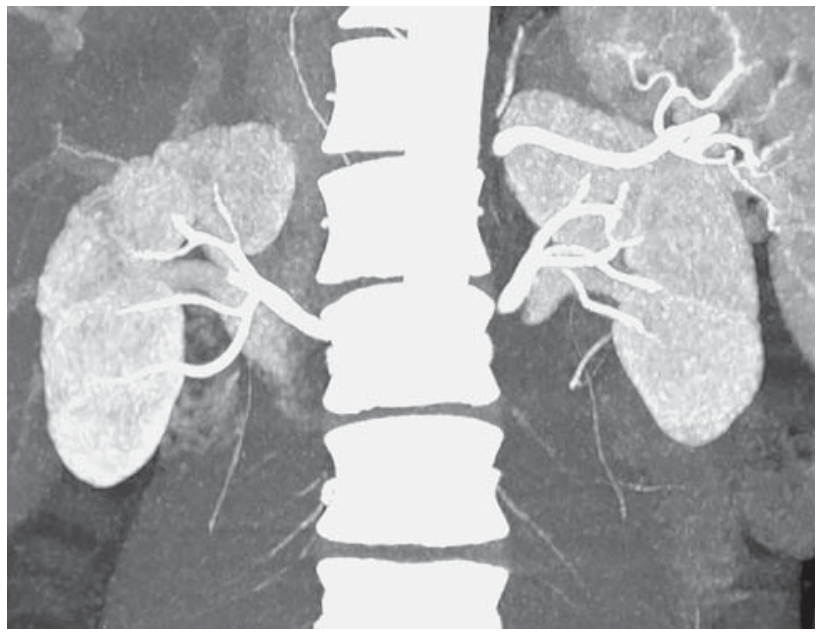

Рис. 4. КТ, артериальная фаза болюсного исследования, корональная плоскость. Справа отмечается рассыпной тип строения почечной артерии.

Fig. 4. СТ, arterial phase of a bolus enhanced study, coronal plane. On the right there is a rasping type of structure of the renal artery.

грамма "Автоплан" для сегментации, моделирования и интраоперационной навигации, которая была разработана в Центре прорывных исследований СамГМУ "Информационные технологии в медицине" (руководитель - профессор А.В. Колсанов) [9].

Технология получения виртуальной 3D-модели практически полностью автоматизирована: "Автоплан" проводит автоматическое выделение и построение изображения почек путем одного "клика" на основе ранее проанализированных и загруженных в базу изображений. Сегментация проводится путем сравнения почек со "средней” моделью с последующим ручным исправлением неточностей. Далее проводили полуавтоматическую сегментацию артерий, вен и ЧЛС с мочеточниками на основании алгоритма сосудистой сегментации путем задания точки отсчета в просвет необходимого органа. Для этого используется специальный алгоритм, учитывающий не только плотность, но и текстуру и поверхность структуры. Совмещение артериальной и выделительной фаз происходит в автоматическом режиме путем наложения на артериальную фазу недостающей информации из выделительной фазы. На основании сегментаций отдельных структур на 2D-изображениях получают полигональную 3D-модель в формате scn с возможностью перевода в stl для 3D-печати. Далее для анализа врачу-рентгенологу представляется полученная интегрированная модель, которую можно было вращать и изменять прозрачность для более точной оценки соотношения ЧлС и сосудов. Среднее время получения модели составило 20 мин.

Выявлено, что артерия нижнего полюса деформирует лоханку и чашечки нижнего полюса, вызывая обструкцию (рис. 5).

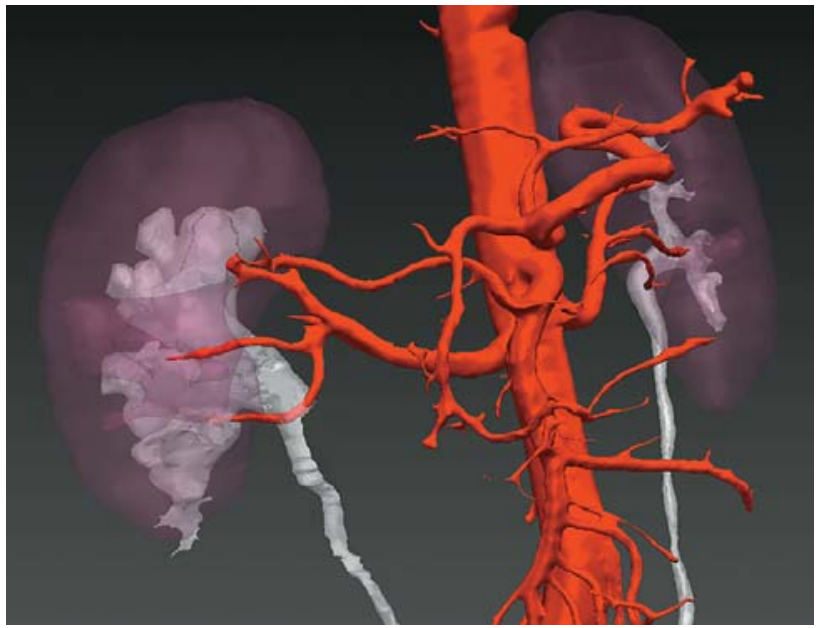

Рис. 5. 3D-реконструкция KT. Справа отмечается перекрест нижнеполюсной артерии и мочеточника. Дистальнее диаметр мочеточника нормальный.

Fig. 5. 3D-reconstruction of CT scan. The junction of the lower pole artery and the ureter is noted on the right. Distant diameter of the ureter is normal.

\section{Клинический пример 3}

Пациент С. обратился с жалобами на почечную колику слева, рецидивирующие пиелонефриты. В анамнезе умеренное расширение лоханки и мочеточника слева, камень лоханки и нижней группы чашечек. Пациенту выполнено сплит-болюсное исследование: 20 мл раствора йогексола 350 мг/мл, через 10 мин введено 80 мл йогексола 350 мг/мл со скоростью 4 мл/с. При анализе изображений выявлено, что наружная подвздошная артерия деформирует мочеточник, вызывая его расширение выше уровня перекреста (рис. 6,7 ).

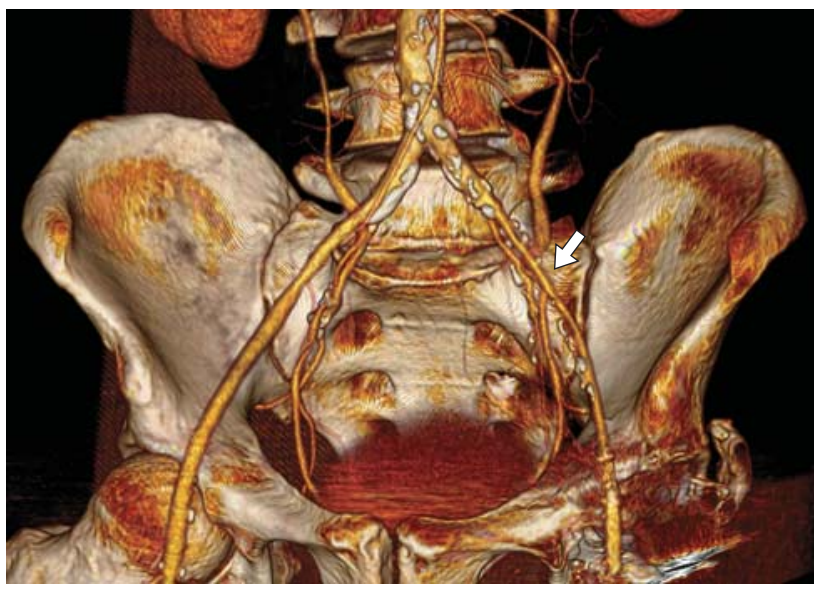

Рис. 6. КТ, артериальная фаза сплит-болюсного исследования, 3D-volume rendering. Слева отмечается перекрест наружной подвздошной артерии и мочеточника.

Fig. 6. СТ, arterial phase of a split-bolus contrast-enhanced study, 3D-volume rendering. On the left, there is a crossing of the external iliac artery and ureter. 


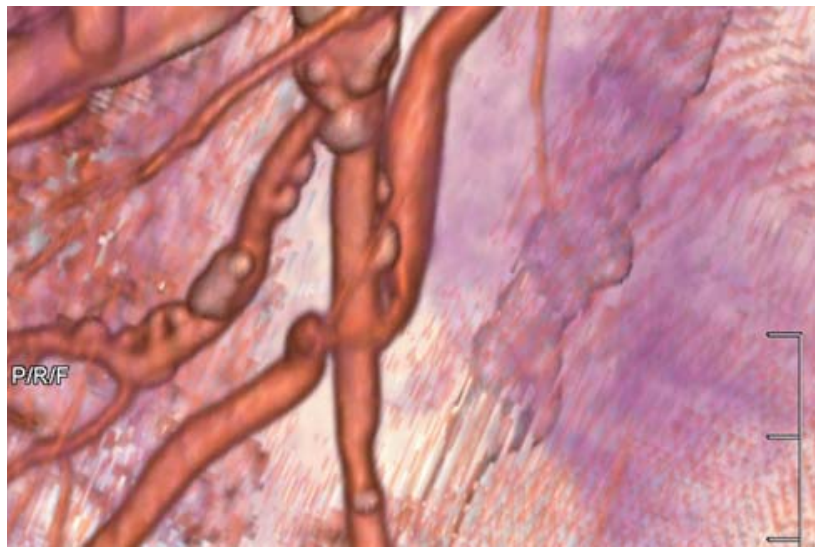

Рис. 7. КТ, артериальная фаза сплит-болюсного исследования, 3D-volume rendering (приближенное изображение). Перекрест наружной подвздошной артерии и мочеточника, отмечается расширение мочеточника проксимальнее перекреста.

Fig. 7. CT, arterial phase of a split-bolus contrast-enhanced study, 3D-volume rendering (approximate image). The intersection of the external iliac artery and ureter, the ureter is dilatated before the intersection.

\section{Обсуждение}

Таким образом, показаны два способа диагностики уретеровазального конфликта при КТ. Сплит-болюсное введение контрастного вещества - удобная методика, которая позволяет сокращать лучевую нагрузку путем исключения из протокола отсроченной фазы. При этом она обладает рядом преимуществ перед стандартным протоколом: изображения позволяют наглядно представить взаимоотношение структур ворот почки на одной фазе. Однако выполнение методики исследования не является устоявшимся. На наш взгляд, в случае достоверного исключения мочекаменной болезни (например, в случае ранее выполненного нативного бесконтрастного исследования) возможно проводить подобное исследование совсем без нативной фазы: пациенту во время ожидания исследования вводят небольшой объем контрастного вещества и через 10 мин проводится стандартное исследование без выделительной фазы. Такая методика ускорит выведение контрастного вещества из-за активного положения пациента и позволит сократить время нахождения пациента на ложементе томографа при ожидании накопления контрастного вещества в ЧлС.

3D-моделирование путем сегментации в области урологии описано, например, в работе Ю.Г. Аляева и соавт. [10]. Авторами показано, что моделирование опухолей почек влияет на объективные числовые показатели вмешательства: объем кро- вопотери и время операции. Моделирования же именно СЛМК до настоящего момента не проводилось.

\section{Заключение}

Учитывая показанную наглядность, мы можем рекомендовать методику 3D-моделирования на основании сегментации после выполнения стандартного протокола КТ с контрастированием. При этом при подозрении на обструкции мочевых путей желательно использовать протокол сплитболюсного введения.

\section{Список литературы}

1. Гудков А.В., Пугачев А.Г. Сосудисто-чашечнолоханочные конфликты. М.: Медицина, 2005. 193 с.

2. Аляев Ю.Г., Ахвледиани Н.Д. Современное применение компьютерной томографии в урологии. Медицинский вестник Башкортостана. 2011; 6 (2): 208-211.

3. Гудков А.В., Пугачев А.Г. Сосудисто-чашечно-лоханочные конфликты. М.: Медицина, 2007. 128 с.

4. Карпенко В.С. Причина гидронефроза и выбор метода оперативного лечения. Урология. 2002; 3 (3): 43-46.

5. Айвазян А.В., Войно-Ясенецкий А.М. Пороки развития почек и мочеточников. М.: Наука, 1988. 448 с.

6. Сизонов В.В. Транспозиция "конфликтного" сосуда при обструкции пиелоуретерального сегмента у детей. Урология. 2009; 4 (4): 51-55.

7. Chow L.C., Sommer F.G. Multidetector CT urography with abdominal compression and three-dimensional reconstruction. Am. J. Roentgenol. 2001; 177: 849-855. DOI: 10.2214/ajr.177.4.1770849.

8. Sauer B., Flocquet M., Batch T., Blum A., Hubert J. Vascular Renal Anatomy and the Ureteropelvic Junction: Preoperative Multidetector CT Scanning with Split-Bolus Injection as a Predictor of Laparoscopic Findings. J. Endourol. 2008; 22 (1): 13-18. http://doi.org/10.1089/end.2006.9857.

9. Колсанов А.В., Манукян А.А., Зельтер П.М., Чаплыгин С.С., Капишников А.В. Виртуальное моделирование операции на печени на основе данных компьютерной томографии. Анналы хирургической гепатологии. 2016; 21 (4): 16-22.

10. Аляев Ю.Г., Фиев Д.Н., Петровский Н.В., Хохлачев С.Б. Использование интраоперационной навигации при органосохраняющих хирургических вмешательствах по поводу опухоли почки. Онкоурология. 2012; 8 (3): 31-37.

\section{References}

1. Gudkov A.V., Pugachev A.G. Vascular-ureter conflicts. M.: Medicine, 2005. 193 p. (In Russian)

2. Alyaev Yu.G., Akhvlediani N.D. Modern application of computed tomography in urology. Bashkortostan Medical Journal. 2011; 6 (2): 208-211. (In Russian)

3. Gudkov A.V., Pugachev A.G. Vascular-ureter conflicts. M.: Medicine, 2007. 128 p. (In Russian)

4. Karpenko V.S. The cause of hydronephrosis and the choice of surgical treatment. Urology. 2002; 3 (3): 43-46. (In Russian)

5. Ayvazyan A.V., Voyno-Yasenetsky A.M. Malformations of the kidneys and ureters. M.: Science, 1988. 448 p. (In Russian) 
6. Sizonov V.V. Transposition of the "conflict" vessel with obstruction of the pyeloureteral segment in children. Urology. 2009; 4 (4): 51-55. (In Russian)

7. Chow L.C., Sommer F.G. Multidetector CT urography with abdominal compression and three-dimensional reconstruction. Am. J. Roentgenol. 2001; 177: 849-855. DOI: 10.2214/ajr.177.4.1770849.

8. Sauer B., Flocquet M., Batch T., Blum A., Hubert J. Vascular Renal Anatomy and the Ureteropelvic Junction: Preoperative Multidetector CT Scanning with Split-Bolus Injection as a Predictor of Laparoscopic Findings.
J. Endourol. 2008; 22 (1): 13-18. http://doi.org/10.1089/end.2006.9857.

9. Kolsanov A.V., Manukyan A.A., Zelter P.M., Chaplygin S.S., Kapishnikov A.V. Virtual simulation of a liver surgery based on computed tomography data. Annaly khirurgicheskoy gepatologii = Annals of HPB surgery. 2016; 21 (4): 16-22. (In Russian)

10. Alyaev Yu.G., Fiev D.N., Petrovsky N.V., Khokhlachev S.B. Use of intraoperative navigation in organ-preserving surgical interventions for kidney tumors. Oncourology. 2012; 8 (3): 31-37. (In Russian)

Для корреспонденции*: Зельтер Павел Михайлович - Россия, Самара, Карла Маркса 165Б. Тел.: +7-917-942-12-34. E-mail: pzelter@mail.ru Зельтер Павел Михайлович - канд. мед. наук, заведующий рентгенологическим отделением клиник СамГМу, ассистент кафедры лучевой диагностики и лучевой терапии с курсом медицинской информатики СамГМУ, Самара.

Соловов Дмитрий Вячеславович - врач-рентгенолог рентгеновского отделения клиник СамГМУ, ассистент кафедры лучевой диагностики и лучевой терапии с курсом медицинской информатики СамГМУ, Самара.

Колсанов Александр Владимирович - доктор мед. наук, профессор, заведующий кафедрой оперативной хирургии и клинической анатомии с курсом инновационных технологий СамГМУ, Самара.

Капишников Александр Викторович - доктор мед. наук, заведующий кафедрой лучевой диагностики и лучевой терапии с курсом медицинской информатики СамГМУ, Самара.

Пекшева Марина Сергеевна - врач-рентгенолог рентгеновского отделения Санкт-Петербургской больницы Святой Елизаветы, СанктПетербург.

Contact*: Pavel M. Zelter - K. Marks str., 165B, Samara, Russia. Phone: +7-917-942-12-34. E-mail: pzelter@mail.ru

Pavel M. Zelter - cand. of med. sci., Head of radiology department of Clinics of Samara Medical State University, Samara.

Dmitrii V. Solovov - radiologist of radiology department of Clinics of Samara Medical State University, assistant of the chair of radiology of Samara Medical State Univer, Samara.

Aleksandr V. Kolsanov - doct. of med. sci., Head of the chair of operative surgery and clinical anatomy of Samara Medical State University, Samara. Aleksandr V. Kapishnikov - doct. of med. sci., Head of the chair of radiology of Samara Medical State University, Samara.

Marina S. Peksheva - radiologist of radiological department of Saint-Petersburg St. Elisabeth state hospital, Saint-Petersburg. 